How are we training the whole dental team? How are we training the whole dental team? How are we training the whole dental team? How are we training the whole dental team? How are we training the whole dental team? How are we training the whole dental team? How are we training the whole dental team? How are we training the whole dental team? How are we training the whole dental team? How are we training the whole dental team? How are we training the whole dental team? How are we training the whole dental team? How are we training the whole dental team? How are we training the whole dental team? How are we training the whole dental team? How are we training the whole dental team? How are we training the whole dental team? How are we training the whole dental team? How are we training the whole dental team? How are we training the whole dental team? How are we training the whole dental team? How are we training the whole dental team? How are we training the whole dental team? How are we training the whole dental team?

Dr Richard Cure* and Professor Robert Ireland* discuss the benefits of including dental practice managers and receptionists in dental team training.
* Postgraduate Dental Education Unit, Institute of Clinical Education, Warwick Medical School

Email: richard.cure@warwick.ac.uk

\section{Rapid expansion}

Not surprisingly, over the last year or so, the registration of dental nurses has been one of the main areas of focus with respect to the development of the training of the dental team. In addition there has been a rapid expansion in the clinical members of the team with the introduction of training programmes for the recently introduced orthodontic therapists and clinical dental technicians and the development of more dental therapist training courses. Combined with this, there has been a significant increase in the number of undergraduate dental students being trained in response to meeting the public demand for better access to primary care dentistry.

\section{Non-clinical DCPs}

It is easy to see with all this clinical training hyperactivity that the non-clinical members of the dental team can be easily overlooked. However, the skills and knowledge of both dental receptionists and practice managers are fundamental to a successful and efficient practice. Receptionists require many unique skills but three of them are fundamental to any successful practice:
- They usually provide the first and last 'person contact' for all patients visiting the practice so their communication skills are vital

- Maintaining and planning the appointment system is the key to clinical efficiency and time management

- A clinical knowledge of the scope and limitations of the services provided is essential in dealing promptly and efficiently with the many patient queries by telephone or direct contact.

Practice managers are less visible to the patients but certainly no less important. They invariably hold responsibility for coordinating the whole dental team. They also make sure that the demands of the clinical and nonclinical aspects of the practice are mutually self-supporting. This requires a comprehensive knowledge not only of current clinical practice, but an appreciation of how new management developments and technological advances can meet the changing needs and demands of the patients thereby helping to retain existing patients' as well as attracting new ones. 
progressing to full-time receptionist and then to practice manager with many of the additional skills necessary being acquired either by bitter experience or by improvisation: this can often result in an inefficient use of both clinical and non-clinical time, or patient dissatisfaction, or both. Is it not time that receptionist and practice manager training is linked to that of the whole dental team so that there is mutual respect and understanding for the roles and contribution that each individual member makes?

'In a world of
fast developing
technology and
the complexity
of constantly
evolving
regulations, it
is essential that
every dental
team member
is up-to-date.'

\section{Primary training}

Opportunities exist for colleges and universities to provide primary training programmes, locally delivered and combined with workplace activity that could lead to professionally recognised qualifications at certificate or diploma level. These could be delivered in conjunction with aspects of the dental nurse, hygienist, therapist and orthodontic therapist courses where there are common core subjects such as communication skills, patient management, and health and safety. An established career pathway recognised by the profession would allow progression from the non-clinical to clinical pathways and vice versa with the potential for the acceptance of accredited prior learning to make this transition more acceptable and efficient.

\section{Personal development plans}

Just because CPD is not compulsory for receptionists and practice managers, it doesn't mean that it's not important. On the contrary, in a world of fast developing technology and the complexity of constantly evolving regulations such as health and safety, data protection, and disability legislation, it is essential that every dental team member is fully informed and up-to-date. Personal development plans (PDPs) as a mechanism by which educational needs are prioritised together with a commitment to address them have long been a part of a dentist's CPD; these need to be developed and structured with the same degree of commitment for the non-clinical dental team specifically at the non-clinical members of the dental team. These are currently provided as part of the activities of the British Dental Practice Managers' Association (www.bdpma. org.uk) with seminars and an excellent quarterly magazine, Dental Management (formerly Networking). However, the lack of a national association for dental receptionists fails to provide a focus for such important ongoing development. This deficiency in training, support, and CPD development needs to be addressed as a matter of some urgency

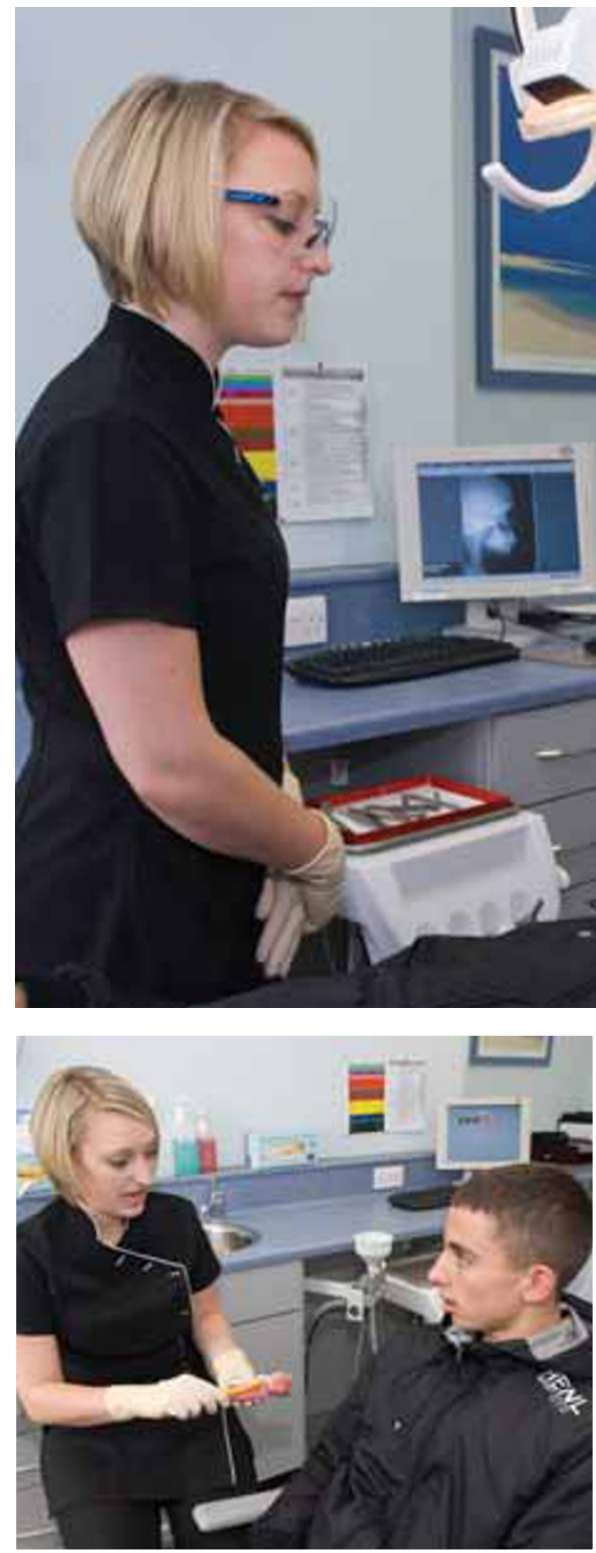

members so that they meet the needs of both the individual and the practice.

\section{Valuing the whole team}

Setting learning objectives is not an academic exercise but should clearly define the outcomes of the learning process and form the core structure of any ongoing PDP. This involves the delivery of post qualification courses and integrated educational programmes targeted
Images from left to right, top to bottom: left-hand page - Education for the whole team; giving information to telephone enquirers. Right-hand page - Clinical teamwork; patient education 